Теорія Ймовір. та Матем. Статист. Вип. 76, 2007
Theor. Probability and Math. Statist.

No. 76, 2008, Pages 131-139

S 0094-9000(08)00737-0

Article electronically published on July 16, 2008

\title{
EXISTENCE AND UNIQUENESS OF THE SOLUTION OF A STOCHASTIC DIFFERENTIAL EQUATION, DRIVEN BY FRACTIONAL BROWNIAN MOTION WITH A STABILIZING TERM
} UDC 519.21

\author{
YU. S. MISHURA AND S. V. POSASHKOV
}

\begin{abstract}
A stochastic differential equation driven by a Wiener process and fractional Brownian motion is considered in the paper. We prove the existence and uniqueness of the solution if the equation contains a certain stabilizing term.
\end{abstract}

\section{INTRODUCTION}

Consider the following stochastic equation written in integral form:

$$
\begin{aligned}
X_{t}= & X_{0}+\int_{0}^{t} a\left(s, X_{s}\right) d s+\int_{0}^{t} b\left(s, X_{s}\right) d W_{s}+\int_{0}^{t} c\left(s, X_{s}\right) d B_{s}^{H} \\
& +\varepsilon \int_{0}^{t} c\left(s, X_{s}\right) d V_{s}, \quad t \in[0, T],
\end{aligned}
$$

where $a, b, c:[0, T] \times \mathbb{R} \rightarrow \mathbb{R}$ are measurable functions, $V$ and $W$ are independent standard Brownian motions, $\varepsilon>0$, and $B^{H}$ is a fractional Brownian motion independent of $W$ and $V$ with the Hurst parameter $H \in(3 / 4,1]$. We assume that the coefficients of this equation are such that all stochastic integrals on the right hand side of (1.1) are well defined.

The integral $\varepsilon \int_{0}^{t} c\left(s, X_{s}\right) d V_{s}$ in (1.1) is considered as a stabilizing term. This term allows us to prove the existence and uniqueness of the solution adapted to the flow $\mathcal{F}_{t}^{\prime}$, $t \geq 0$, where

$$
\mathcal{F}_{t}^{\prime}=\sigma\left\{X_{0}, W_{s},\left(\varepsilon V_{s}+B_{s}^{H}\right) \mid s \in[0, t]\right\} .
$$

The results of this paper remain true for the case of $b=0$. The case of $b=0$ and $\varepsilon=0$ corresponds to the equation containing a fractional Brownian motion only (an equation without a standard Brownian motion). Conditions for the existence and uniqueness of a solution for the latter equation are considered in the paper [1. One of these conditions is that the derivative $\partial_{x} c(s, x)$ exists. We avoid this and some other conditions by introducing a stabilizing term. Conditions for the existence and uniqueness of a solution of a semilinear equation with $\varepsilon=0, b(s, x)=b x$, and $c(s, x)=c x$ are obtained in [2].

2000 Mathematics Subject Classification. Primary 60G15; Secondary 60H05, 60H10.

Key words and phrases. Stochastic differential equation, existence and uniqueness of the solution, fractional Brownian motion.

The research of the first coauthor is partially supported by the NATO grant PST.CLG 890408. 


\section{MAIN PART}

2.1. Existence and uniqueness of solutions of some auxiliary equations. According to the paper [3], the process $M_{t}^{H, \varepsilon}:=\varepsilon V_{t}+B_{t}^{H}$ considered with respect to the flow $\mathcal{F}_{t}, t \in[0, T]$, where

$$
\mathcal{F}_{t}=\sigma\left\{\varepsilon V_{s}+B_{s}^{H}, s \in[0, t]\right\},
$$

is equivalent to the standard Brownian motion. Since $X_{0}, W, B^{H}$, and $V$ are independent, this implies that the process $M_{t}^{H, \varepsilon}$ is equivalent to the standard Brownian motion with respect to the flow $\mathcal{F}_{t}^{\prime}$. Using Hitsuda's results [4, one can represent this process in the following form:

$$
\begin{aligned}
M_{t}^{H, \varepsilon} & =\varepsilon\left(V_{t}+\frac{1}{\varepsilon} B_{t}^{H}\right) \\
& =\varepsilon\left(V_{t}^{\prime}+\int_{0}^{t} \int_{0}^{s} r_{\varepsilon}(s, u) d V_{u}^{\prime} d s\right) \\
& =\varepsilon V_{t}^{\prime}+\int_{0}^{t} \int_{0}^{s} \varepsilon r_{\varepsilon}(s, u) d V_{u}^{\prime} d s,
\end{aligned}
$$

where $V^{\prime}$ is another standard Brownian motion adapted to the flow $\mathcal{F}_{t}\left(\mathcal{F}_{t}^{\prime}\right)$ and where $r_{\varepsilon} \in \mathbf{L}^{2}\left([0, T]^{2}\right)$ is a corresponding kernel dependent on $\varepsilon$ and $H$.

Using (2.1) we rewrite equation (1.1) as follows:

$$
\begin{aligned}
X_{t}= & X_{0}+\int_{0}^{t} a\left(s, X_{s}\right) d s+\int_{0}^{t} b\left(s, X_{s}\right) d W_{s}+\varepsilon \int_{0}^{t} c\left(s, X_{s}\right) d V_{s}^{\prime} \\
& +\int_{0}^{t} c\left(s, X_{s}\right) \int_{0}^{s} \varepsilon r_{\varepsilon}(s, u) d V_{u}^{\prime} d s .
\end{aligned}
$$

Let

$$
R_{t}^{\varepsilon}:=\int_{0}^{t}\left(\int_{0}^{s} \varepsilon r_{\varepsilon}(s, u) d V_{u}^{\prime}\right)^{2} d s, \quad t \in[0, T] .
$$

Define a sequence of stopping times $\tau_{N}, N \in \mathbb{N}$, by

$$
\tau_{N}:=\inf \left\{t \in[0, T]: R_{t}^{\varepsilon}>N\right\} \wedge T .
$$

We set $\tau_{N}:=T$ if $\left\{t \in[0, T]: R_{t}^{\varepsilon}>N\right\}=\varnothing$.

Now we prove that $\tau_{N} \rightarrow T$ with probability one.

Lemma 2.1. Let a process $R_{t}^{\varepsilon}, \varepsilon>0, t \in[0, T]$, and stopping times $\tau_{N}, N \in \mathbb{N}$, be defined by (2.3) and (2.4), respectively. Then for all $\varepsilon>0$,

$$
\tau_{N} \rightarrow T
$$

with probability one.

Proof. We evaluate the probability of the event that $\tau_{N}<T$ :

$$
\begin{aligned}
\mathrm{P}\left\{\tau_{N}<T\right\} & =\mathrm{P}\left\{\int_{0}^{T}\left(\int_{0}^{s} \varepsilon r_{\varepsilon}(s, u) d V_{u}^{\prime}\right)^{2} d s>N\right\} \\
& \leq \frac{\varepsilon^{2}}{N} \mathrm{E} \int_{0}^{T}\left(\int_{0}^{s} r_{\varepsilon}(s, u) d V_{u}^{\prime}\right)^{2} d s \\
& =\frac{\varepsilon^{2}}{N} \int_{0}^{T} \int_{0}^{s} r_{\varepsilon}^{2}(s, u) d u d s \\
& \leq \frac{\varepsilon^{2}}{N} \int_{0}^{T} \int_{0}^{T} r_{\varepsilon}^{2}(s, u) d u d s \leq \frac{\varepsilon^{2} K_{\varepsilon}}{N},
\end{aligned}
$$


where

$$
K_{\varepsilon}=\left\|r_{\varepsilon}\right\|_{\mathbf{L}^{2}\left([0, T]^{2}\right)}^{2}<\infty .
$$

Thus $\mathrm{P}\left\{\tau_{N}<T\right\} \rightarrow 0$ as $N \rightarrow \infty$, whence Lemma 2.1 follows, since $\tau_{N}$ are nondecreasing in $N$.

Remark 2.2. We proved, in fact, that for all $\omega \in \Omega_{0}$ where the event $\Omega_{0}$ is such that $\mathrm{P}\left(\Omega_{0}\right)=1$, there exists a number $N=N(\omega)$ for which $\tau_{N(\omega)}=T$.

Without loss of generality we assume that $\varepsilon=1$ and $r(t, s):=r_{1}(t, s)$ in what follows. Consider the following family of equations:

$$
\begin{aligned}
X_{t}^{N}= & X_{0}+\int_{0}^{t} a\left(s, X_{s}^{N}\right) d s+\int_{0}^{t} b\left(s, X_{s}^{N}\right) d W_{s}+\int_{0}^{t} c\left(s, X_{s}^{N}\right) d V_{s}^{\prime} \\
& +\int_{0}^{t \wedge \tau_{N}} c\left(s, X_{s}^{N}\right) \int_{0}^{s} r(s, u) d V_{u}^{\prime} d s .
\end{aligned}
$$

We prove that the solution $X_{t}^{N}$ of equation (2.5) exists for all $N \in \mathbb{N}$.

Theorem 2.3. Assume that

1. The functions $a(\cdot, 0), b(\cdot, 0)$, and $c(\cdot, 0)$ are bounded by some constant $L$ in the interval $[0, T]$.

2. There exists an increasing function $l(s):[0, T] \rightarrow \mathbb{R}$ such that

$$
\begin{aligned}
& (a(s, x)-a(s, y))^{2}+(b(s, x)-b(s, y))^{2}+(c(s, x)-c(s, y))^{2} \\
& \quad \leq l(s)(x-y)^{2}
\end{aligned}
$$

for all $x, y \in \mathbb{R}$.

If $X_{0}$ is $\mathcal{F}_{0}^{\prime}$-measurable and $\mathrm{E} X_{0}^{2}<\infty$, then equation (2.5) possesses an $\mathcal{F}_{t}^{\prime}$-measurable solution $X_{t}^{N}$ such that $\mathrm{E}\left(X_{t}^{N}\right)^{2}<\infty$.

Proof. Following the idea of the proof of Theorem 3 in Chapter 4, Section 1 of [5], we construct the solution by successive approximations. Put $X_{t}^{N, 0}:=X_{0}$ and

$$
\begin{aligned}
X_{t}^{N, n+1}= & X_{0}+\int_{0}^{t} a\left(s, X_{s}^{N, n}\right) d s+\int_{0}^{t} b\left(s, X_{s}^{N, n}\right) d W_{s}+\int_{0}^{t} c\left(s, X_{s}^{N, n}\right) d V_{s}^{\prime} \\
& +\int_{0}^{t \wedge \tau_{N}} c\left(s, X_{s}^{N, n}\right) \int_{0}^{s} r(s, u) d V_{u}^{\prime} d s .
\end{aligned}
$$

If $X_{t}^{N, n}$ is defined for some $n$, is $\mathcal{F}_{t}^{\prime}$-measurable, and $\mathrm{E}\left(X_{t}^{N, n}\right)^{2}$ is bounded on [0,T], then the integrals on the right hand side of (2.7) are well defined and are $\mathcal{F}_{t}^{\prime}$-measurable. Now we prove that $\mathrm{E}\left(X_{t}^{N, n+1}\right)^{2}$ is bounded in $[0, T]$. Indeed,

$$
\begin{aligned}
\left(X_{t}^{N, n+1}-X_{t}^{N, 0}\right)^{2} \leq & 4\left(\int_{0}^{t} a\left(s, X_{s}^{N, n}\right) d s\right)^{2}+4\left(\int_{0}^{t} b\left(s, X_{s}^{N, n}\right) d W_{s}\right)^{2} \\
& +4\left(\int_{0}^{t} c\left(s, X_{s}^{N, n}\right) d V_{s}^{\prime}\right)^{2} \\
& +4\left(\int_{0}^{t \wedge \tau_{N}} c\left(s, X_{s}^{N, n}\right) \int_{0}^{s} r(s, u) d V_{u}^{\prime} d s\right)^{2}
\end{aligned}
$$




$$
\begin{gathered}
\mathrm{E}\left(\int_{0}^{t} a\left(s, X_{s}^{N, n}\right) d s\right)^{2} \leq T \int_{0}^{t} \mathrm{E}\left(a\left(s, X_{s}^{N, n}\right)\right)^{2} d s \\
\leq 2 T \int_{0}^{t} a^{2}(s, 0) d s+2 T \int_{0}^{t} \mathrm{E}\left(a\left(s, X_{s}^{N, n}\right)-a(s, 0)\right)^{2} d s \\
\leq 2(L T)^{2}+2 T l(T) \int_{0}^{T} \mathrm{E}\left(X_{s}^{N, n}\right)^{2} d s \\
\mathrm{E}\left(\int_{0}^{t} b\left(s, X_{s}^{N, n}\right) d W_{s}\right)^{2} \leq 2 \int_{0}^{t} b^{2}(s, 0) d s+2 \int_{0}^{t} \mathrm{E}\left(b\left(s, X_{s}^{N, n}\right)-b(s, 0)\right)^{2} d s \\
\leq 2 T L^{2}+2 l(T) \int_{0}^{T} \mathrm{E}\left(X_{s}^{N, n}\right)^{2} d s .
\end{gathered}
$$

Similarly,

$$
\mathrm{E}\left(\int_{0}^{t} c\left(s, X_{s}^{N, n}\right) d V_{s}^{\prime}\right)^{2} \leq\left(2 T L^{2}+2 l(T) \int_{0}^{T} \mathrm{E}\left(X_{s}^{N, n}\right)^{2} d s\right) .
$$

By the definition of the stopping times $\tau_{N}$ we have

$$
\begin{aligned}
& \mathrm{E}\left(\int_{0}^{t \wedge \tau_{N}} c\left(s, X_{s}^{N, n}\right) \int_{0}^{s} r(s, u) d V_{u}^{\prime} d s\right)^{2} \\
& \quad \leq \mathrm{E}\left[\int_{0}^{t \wedge \tau_{N}}\left(c\left(s, X_{s}^{N, n}\right)\right)^{2} d s \int_{0}^{t \wedge \tau_{N}}\left(\int_{0}^{s} r(s, u) d V_{u}^{\prime}\right)^{2} d s\right] \\
& \quad \leq N \mathrm{E}\left(\int_{0}^{t} c\left(s, X_{s}^{N, n}\right) d s\right)^{2} \leq N\left(2(L T)^{2}+2 T l(T) \int_{0}^{T} \mathrm{E}\left(X_{s}^{N, n}\right)^{2} d s\right)
\end{aligned}
$$

in view of Hölder's inequality. The latter bounds imply that there exists a constant $C_{N}$ such that

$$
\mathrm{E}\left(X_{t}^{N, n+1}\right)^{2} \leq C_{N}\left(\mathrm{E} X_{0}^{2}+\int_{0}^{t} \mathrm{E}\left(X_{s}^{N, n}\right)^{2} d s+L^{2}\right) .
$$

Since the process $X_{0}$ is $\mathcal{F}_{t}^{\prime}$-measurable and $\mathrm{E}\left(X_{t}^{N, 0}\right)^{2}=\mathrm{E} X_{0}^{2}$, the processes $X_{t}^{N, n}$ are well defined for all $n$, are $\mathcal{F}_{t}^{\prime}$-measurable, and the second moments $\mathrm{E}\left(X_{t}^{N, n}\right)^{2}$ are bounded.

Further,

$$
\begin{aligned}
\mathrm{E}\left(X_{t}^{N, n+1}-X_{t}^{N, n}\right)^{2} \leq & 4 \mathrm{E}\left(\int_{0}^{t}\left(a\left(s, X_{s}^{N, n}\right)-a\left(s, X_{s}^{N, n-1}\right)\right) d s\right)^{2} \\
& +4 \mathrm{E}\left(\int_{0}^{t}\left(b\left(s, X_{s}^{N, n}\right)-b\left(s, X_{s}^{N, n-1}\right)\right) d W_{s}\right)^{2} \\
& +4 \mathrm{E}\left(\int_{0}^{t}\left(c\left(s, X_{s}^{N, n}\right)-c\left(s, X_{s}^{N, n-1}\right)\right) d V_{s}^{\prime}\right)^{2} \\
& +4 \mathrm{E}\left(\int_{0}^{t \wedge \tau_{N}}\left(c\left(s, X_{s}^{N, n}\right)-c\left(s, X_{s}^{N, n-1}\right)\right) \int_{0}^{s} r(s, u) d V_{u}^{\prime} d s\right)^{2} .
\end{aligned}
$$

Using the definition of the stopping times $\tau_{N}$ and condition 2 we prove that there exists a constant $K_{N, T}$ such that

$$
\begin{gathered}
\mathrm{E}\left(X_{t}^{N, n+1}-X_{t}^{N, n}\right)^{2} \leq K_{N, T} \int_{0}^{t} \mathrm{E}\left(X_{s}^{N, n}-X_{s}^{N, n-1}\right)^{2} d s, \quad n \geq 1, \\
\mathrm{E}\left(X_{t}^{N, 1}-X_{t}^{N, 0}\right)^{2} \leq K_{N, T} \int_{0}^{t} \mathrm{E}\left(X_{s}^{N, 0}\right)^{2} d s=K_{N, T} t \mathrm{E} X_{0}^{2}
\end{gathered}
$$


by Hölder's inequality. This implies that

$$
\mathrm{E}\left(X_{t}^{N, n+1}-X_{t}^{N, n}\right)^{2} \leq\left(K_{N, T}\right)^{n+1} \frac{T^{n+1}}{(n+1) !} \mathrm{E} X_{0}^{2} .
$$

Since

$$
\sum_{n \in \mathbb{N}} \sup _{t \in[0, T]}\left(\mathrm{E}\left(X_{t}^{N, n+1}-X_{t}^{N, n}\right)^{2}\right)^{1 / 2}<\infty,
$$

the stochastic process $X_{t}^{N, n}$ converges in the mean square sense to $X_{t}^{N}$ as $n \rightarrow \infty$. Moreover,

$$
\lim _{n \rightarrow \infty} \sup _{t \in[0, T]} \mathrm{E}\left(X_{t}^{N, n}-X_{t}^{N}\right)^{2}=0 .
$$

Note that the process $X_{t}^{N}$ is $\mathcal{F}_{t}^{\prime}$-measurable.

Now we prove that (2.5) holds almost surely. Equality (2.5) holds almost surely if the integrals on the right hand side of (2.7) converge in the mean square sense to the corresponding integrals on the right hand side of (2.5) as $n \rightarrow \infty$. We prove this convergence for the integrals involving the stopping time $\tau_{N}$. The proof for other integrals is the same. We have

$$
\begin{aligned}
& \mathrm{E}\left(\int_{0}^{t \wedge \tau_{N}}\left(c\left(s, X_{s}^{N, n}\right)-c\left(s, X_{s}^{N}\right)\right) \int_{0}^{s} r(s, u) d V_{u}^{\prime} d s\right)^{2} \\
& \quad \leq T N \varepsilon^{2} \int_{0}^{T} \mathrm{E}\left(c\left(s, X_{s}^{N, n}\right)-c\left(s, X_{s}^{N}\right)\right)^{2} d s \\
& \quad \leq T l(T) N \int_{0}^{T} \mathrm{E}\left(X_{s}^{N, n}-X_{s}^{N}\right)^{2} d s
\end{aligned}
$$

The expression on the right hand side approaches zero in view of (2.8).

The following result provides conditions for the uniqueness of a solution of equation (2.5).

Theorem 2.4. Let the coefficients of equation (2.5) be such that, for any $r \geq 0$, there exists a constant $l_{r}$ for which

$$
(a(s, x)-a(s, y))^{2}+(b(s, x)-b(s, y))^{2}+(c(s, x)-c(s, y))^{2} \leq l_{r}(x-y)^{2}
$$

for $|x| \leq r,|y| \leq r$, and $s \leq r$. If an $\mathcal{F}_{t}^{\prime}$-adapted solution of equation (2.5) exists in the interval $[0, T]$, then it is unique.

Proof. Let $X_{t}^{N}$ and $\hat{X}_{t}^{N}$ be two solutions of equation (2.5). For every $r>0$, we consider the stopping times $\xi_{r}:=\inf \left\{t \in[0, T]:\left|X_{t}^{N}\right|+\left|\hat{X}_{t}^{N}\right| \geq r\right\} \wedge r \wedge T$. Then

$$
\begin{aligned}
X_{t \wedge \xi_{r}}^{N}-\hat{X}_{t \wedge \xi_{r}}^{N}= & \int_{0}^{t \wedge \xi_{r}}\left(a\left(s, X_{s}^{N}\right)-a\left(s, \hat{X}_{s}^{N}\right)\right) d s \\
& +\int_{0}^{t \wedge \xi_{r}}\left(b\left(s, X_{s}^{N}\right)-b\left(s, \hat{X}_{s}^{N}\right)\right) d W_{s} \\
& +\int_{0}^{t \wedge \xi_{r}}\left(c\left(s, X_{s}^{N}\right)-c\left(s, \hat{X}_{s}^{N}\right)\right) d V_{s}^{\prime} \\
& +\int_{0}^{t \wedge \xi_{r} \wedge \tau_{N}}\left(c\left(s, X_{s}^{N}\right)-c\left(s, \hat{X}_{s}^{N}\right)\right) \int_{0}^{s} r(s, u) d V_{u}^{\prime} d s .
\end{aligned}
$$


Using the properties of stochastic integrals, Hölder's inequality, and boundedness of the second moments of $\xi_{r}$, we obtain that

$$
\begin{aligned}
& \mathrm{E}\left(\int_{0}^{t \wedge \xi_{r}}\left(a\left(s, X_{s}^{N}\right)-a\left(s, \hat{X}_{s}^{N}\right)\right) d s\right)^{2} \leq T l_{r} \mathrm{E} \int_{0}^{t \wedge \xi_{r}}\left(X_{s}^{N}-\hat{X}_{s}^{N}\right)^{2} d s, \\
& \mathrm{E}\left(\int_{0}^{t \wedge \xi_{r}}\left(b\left(s, X_{s}^{N}\right)-b\left(s, \hat{X}_{s}^{N}\right)\right) d W_{s}\right)^{2} \leq l_{r} \mathrm{E} \int_{0}^{t \wedge \xi_{r}}\left(X_{s}^{N}-\hat{X}_{s}^{N}\right)^{2} d s, \\
& \mathrm{E}\left(\int_{0}^{t \wedge \xi_{r}}\left(c\left(s, X_{s}^{N}\right)-c\left(s, \hat{X}_{s}^{N}\right)\right) d W_{s}\right)^{2} \leq l_{r} \mathrm{E} \int_{0}^{t \wedge \xi_{r}}\left(X_{s}^{N}-\hat{X}_{s}^{N}\right)^{2} d s
\end{aligned}
$$

and

$$
\begin{aligned}
& \mathrm{E}\left(\int_{0}^{t \wedge \xi_{r} \wedge \tau_{N}}\left(c\left(s, X_{s}^{N}\right)-c\left(s, \hat{X}_{s}^{N}\right)\right) \int_{0}^{s} r(s, u) d V_{u}^{\prime} d s\right)^{2} d s \\
& \quad \leq l_{r} N \mathrm{E} \int_{0}^{t \wedge \xi_{r}}\left(X_{s}^{N}-\hat{X}_{s}^{N}\right)^{2} d s
\end{aligned}
$$

in view of the assumptions of the theorem. Thus

$$
\mathrm{E}\left(X_{t \wedge \xi_{r}}^{N}-\hat{X}_{t \wedge \xi_{r}}^{N}\right)^{2} \leq L_{N, T} \mathrm{E} \int_{0}^{t \wedge \xi_{r}}\left(X_{s}^{N}-\hat{X}_{s}^{N}\right)^{2} d s
$$

for some $L_{N, T}$. This implies that

$$
\mathrm{E}\left(X_{t}^{N}-\hat{X}_{t}^{N}\right)^{2} \mathbb{I}_{\left\{\xi_{r}>t\right\}} \leq L_{N, T} \int_{0}^{t} \mathrm{E}\left(X_{s}^{N}-\hat{X}_{s}^{N}\right)^{2} \mathbb{I}_{\left\{\xi_{r}>s\right\}} d s
$$

Now we obtain from the Gronwall lemma that

$$
\mathrm{E}\left(X_{t}^{N}-\hat{X}_{t}^{N}\right)^{2} \mathbb{I}_{\left\{\xi_{r}>t\right\}}=0 .
$$

Since

$$
\mathbb{I}_{\left\{\xi_{r}>t\right\}} \rightarrow 1 \quad \text { as } r \rightarrow \infty
$$

we have

$$
X_{t}^{N}=\hat{X}_{t}^{N} \quad \text { for } t \in[0, T) .
$$

The solutions are continuous at the point $T$, whence we get that $X_{T}^{N}=\hat{X}_{T}^{N}$. The theorem is proved.

Lemma 2.5. Let the assumptions of Theorem 2.4 hold. We also assume that, for some constant $L$,

$$
(a(s, x))^{2}+(b(s, x))^{2}+(c(s, x))^{2} \leq L\left(1+x^{2}\right) .
$$

If a solution of equation (2.5) exists, then there exists a constant $K$ such that

$$
\mathrm{E}\left(\sup _{s \leq t}\left(X_{s}^{N}\right)^{2} \mid X_{0}\right) \leq K\left(X_{0}^{2}+1\right) e^{K t} .
$$


Proof. We estimate $\mathrm{E}\left(\left(X_{t}^{N}\right)^{2} \mid X_{0}\right)$ :

$$
\begin{aligned}
\mathrm{E}\left(\left(X_{t}^{N}\right)^{2} \mid X_{0}\right) \leq 5 \mathrm{E}\left[X_{0}^{2}\right. & +\left(\int_{0}^{t} a\left(s, X_{s}^{N}\right) d s\right)^{2}+\left(\int_{0}^{t} b\left(s, X_{s}^{N}\right) d W_{s}\right)^{2} \\
& +\left(\int_{0}^{t} c\left(s, X_{s}^{N}\right) d V_{s}^{\prime}\right)^{2} \\
& \left.+\left(\int_{0}^{t \wedge \tau_{N}} c\left(s, X_{s}^{N}\right) \int_{0}^{s} r(s, u) d V_{u}^{\prime} d s\right)^{2} \mid X_{0}\right] \\
\leq 5 \mathrm{E}\left[X_{0}^{2}\right. & +t \int_{0}^{t} a\left(s, X_{s}^{N}\right)^{2} d s+\int_{0}^{t} b\left(s, X_{s}^{N}\right)^{2} d s \\
& \left.+\int_{0}^{t} c\left(s, X_{s}^{N}\right)^{2} d s+N \int_{0}^{t} c\left(s, X_{s}^{N}\right)^{2} d s \mid X_{0}\right] \\
\leq 5\left(X_{0}^{2}+\right. & \left.L(T+1+N) \int_{0}^{t}\left(1+\mathrm{E}\left(\left(X_{s}^{N}\right)^{2} \mid X_{0}\right)\right) d s\right) .
\end{aligned}
$$

Thus the Gronwall lemma implies that

$$
1+\mathrm{E}\left(\left(X_{t}^{N}\right)^{2} \mid X_{0}\right) \leq\left(5 X_{0}^{2}+1\right) e^{5 L(1+N+T) t} .
$$

Using the latter bound, the following inequalities

$$
\begin{gathered}
\mathrm{E}\left(\sup _{s \leq t}\left(\int_{0}^{s} a\left(u, X_{u}^{N}\right) d u\right)^{2} \mid X_{0}\right) \leq t \int_{0}^{t} \mathrm{E}\left(a\left(s, X_{s}^{N}\right)^{2} \mid X_{0}\right) d s \\
\mathrm{E}\left(\sup _{s \leq t}\left(\int_{0}^{s} b\left(u, X_{u}^{N}\right) d W_{u}\right)^{2} \mid X_{0}\right) \leq 4 \int_{0}^{t} \mathrm{E}\left(b\left(s, X_{s}^{N}\right)^{2} \mid X_{0}\right) d s \\
\mathrm{E}\left(\sup _{s \leq t}\left(\int_{0}^{s} c\left(u, X_{u}^{N}\right) d V_{u}^{\prime}\right)^{2} \mid X_{0}\right) \leq 4 \int_{0}^{t} \mathrm{E}\left(c\left(s, X_{s}^{N}\right)^{2} \mid X_{0}\right) d s \\
\mathrm{E}\left(\sup _{s \leq t}\left(\int_{0}^{s \wedge \tau_{N}} c\left(u, X_{u}^{N}\right) \int_{0}^{u} r(u, v) d V_{v}^{\prime} d u\right)^{2} \mid X_{0}\right) \leq N t \int_{0}^{t} \mathrm{E}\left(c\left(s, X_{s}^{N}\right)^{2} \mid X_{0}\right) d s
\end{gathered}
$$

and (2.10) and (2.12) we prove (2.11).

Theorem 2.6. Let the assumptions of Theorem 2.4 hold for the coefficients of equation (2.5). We also assume condition (2.10). If $X_{0}$ is $\mathcal{F}_{0}^{\prime}$-measurable, then equation (2.5) possesses an $\mathcal{F}_{t}^{\prime}$-measurable solution $X_{t}^{N}$ in $[0, T]$.

Proof. Let $\phi_{n}(x)=x$ for $|x|<n$ and $\phi_{n}(x)=n x /|x|$ for $x \geq n$. Also let

$$
a_{n}(t, x)=a\left(t, \phi_{n}(x)\right), \quad b_{n}(t, x)=b\left(t, \phi_{n}(x)\right), \quad b_{n}(t, x)=b\left(t, \phi_{n}(x)\right) .
$$

Denote by $X_{t}^{N n}$ a solution of the equation

$$
\begin{aligned}
X_{t}^{N n}= & \phi_{n}\left(X_{0}\right)+\int_{0}^{t} a_{n}\left(s, X_{s}^{N n}\right) d s+\int_{0}^{t} b_{n}\left(s, X_{s}^{N n}\right) d W_{s} \\
& +\int_{0}^{t} c_{n}\left(s, X_{s}^{N n}\right) d V_{s}^{\prime}+\int_{0}^{t \wedge \tau_{N}} c_{n}\left(s, X_{s}^{N n}\right) \int_{0}^{s} r(s, u) d V_{u}^{\prime} d s .
\end{aligned}
$$

The existence of an $\mathcal{F}_{t}^{\prime}$-measurable solution follows from Theorem2.3 Indeed, condition 1 follows from (2.10), while condition 2 holds for $l(s)=l_{n}$ if $s \leq n$ and for $l(s)=l_{s}$ if 
$s \geq n$. Put $\zeta_{n}=\inf \left\{t \in[0, T]: X_{t}^{N n}>n\right\} \wedge T$. It is easy to see that

$$
X_{t}^{N n_{1}}=X_{t}^{N n}
$$

for $n_{1}>n$ and all $t<\zeta_{n}$. Lemma 2.5 implies that there exists a function $g(t)$ that does not depend on $n$ and such that

$$
\mathrm{E}\left(\sup _{s \leq t}\left(X_{s}^{N n}\right)^{2} \mid X_{0}\right) \leq g(t)\left(1+\phi_{n}^{2}\left(X_{0}\right)\right) .
$$

Thus

$$
\mathrm{P}\left\{\zeta_{n} \leq t \mid X_{0}\right\} \leq \frac{1}{n^{2}} \mathrm{E}\left(\sup _{s \leq t}\left(X_{s}^{N n}\right)^{2} \mid X_{0}\right) \leq \frac{g(t)\left(1+X_{0}^{2}\right)}{n^{2}} .
$$

Since $X_{t}^{N n_{1}}=X_{t}^{N n}$ for $n_{1}>n$ and $t<\zeta_{n}$, we obtain $\zeta_{n_{1}} \geq \zeta_{n}$. Therefore $\zeta_{n} \rightarrow T$ as $n \rightarrow \infty$ almost surely by (2.14). This means that, for all $t \in[0, T)$, there exists a number $n$ such that $\zeta_{n}>t$, whence $X_{t}^{N n_{1}}=X_{t}^{N n}$ for all $n_{1}>n$. Put $X_{t}^{N}:=X_{t}^{N n}$ for $t<\zeta_{n}$. If $t<\zeta_{n}$, then

$$
\begin{aligned}
& a_{n}\left(t, X_{t}^{N n}\right)=a\left(t, X_{t}^{N}\right), \\
& b_{n}\left(t, X_{t}^{N n}\right)=b\left(t, X_{t}^{N}\right), \\
& c_{n}\left(t, X_{t}^{N n}\right)=c\left(t, X_{t}^{N}\right) .
\end{aligned}
$$

Hence $X_{t}^{N}, t \in[0, T)$, satisfies equation (2.5). The solution is extended to the point $T$ by continuity.

Remark 2.7. The assumptions of Theorem 2.6 imply those of Theorem 2.4. Therefore the solution of equation (2.5) is unique.

2.2. Existence and uniqueness of the initial equation. We prove that the assumptions of Theorem 2.6 imply that a solution of equation (1.1) exists, is adapted to the flow $\mathcal{F}_{t}^{\prime}$, and is unique.

Theorem 2.8. Let the assumptions of Theorem 2.6 hold. Then there exists a unique solution of equation (1.1).

Proof. First we note that definition (2.4) of the stopping times $\tau_{N}, N \in \mathbb{N}$, Theorem 2.6. and Remark 2.7 imply for all $N_{1}, N_{2} \in \mathbb{N}$ that solutions $X_{t}^{N_{1}}$ and $X_{t}^{N_{2}}$ coincide in the interval $\left[0, \tau_{N_{1}} \wedge \tau_{N_{2}}\right]$. Then we get from Lemma 2.1 that, for all $t \in[0, T)$, there exists a number $N$ such that

$$
X_{t}^{N_{1}}=X_{t}^{N}
$$

for all $N_{1} \geq N$. We put $X_{t}=X_{t}^{N}$ for $t<\tau_{N}$ and $X_{T}:=X_{T-}$.

Now we show that $X_{t}, t \in[0, T]$, satisfies equation (1.1). Indeed, equations (1.1) and (2.2) are equivalent with respect to the flow $\mathcal{F}_{t}^{\prime}$ and equation (2.5) coincides with equation (2.2) up to the moment $\tau_{N}$. If $N$ is such that $\tau_{N}>t$, then $X_{t}$ satisfies equation (2.5). Thus $X_{t}$ satisfies equation (1.1).

Remark 2.9. Instead of the term $\int_{0}^{t} b\left(s, X_{s}\right) d W_{s}$ one can use an arbitrary finite sum $\sum_{i=1}^{K} \int_{0}^{t} b_{i}\left(s, X_{s}\right) d\left(W_{i}\right)_{s}, K \in \mathbb{N} \cup\{0\}$, in equation (1.1) if standard Brownian motions $W_{i}, i=1, \ldots, K$, are jointly independent and do not depend on both $B^{H}$ and $V$.

Remark 2.10. Equation (1.1) has a unique solution in every finite interval $[0, T]$.

\section{Concluding Remarks}

We considered equation (1.1) driven by fractional Brownian motion containing a certain stabilizing term. We proved the existence and uniqueness of the solution of this equation. 


\section{BIBLIOGRAPHY}

1. D. Nualart and A. Răşcanu, Differential equations driven by fractional Brownian motion, Collection Mathematics 53 (2002), no. 1, 55-81. MR.1893308 (2003f:60105)

2. Yu. Krvavych and Yu. Mishura, Exponential formula and Girsanov theorem for mixed semilinear stochastic differential equations, Mathematical Finance (Trends in Mathematics), Birkhäuser, Basel, 2001, pp. 230-238. MR.1882834

3. P. Cheridito, Regularizing Fractional Brownian Motion with a View towards Stock Price Modelling, Ph.D. Thesis, Swiss Federal Institute of Technology, Zurich, 2001.

4. M. Hitsuda, Representation of Gaussian processes equivalent to Wiener process, Osaka J. Math. 5 (1968), 299-312. MR0243614(39:4935)

5. I. I. Gikhman and A. V. Skorokhod, Stochastic Differential Equations and their Applications, "Naukova dumka", Kiev, 1982. (Russian) MR678374 (84j:60003)

Department of Probability Theory and Mathematical Statistics, Faculty for Mechanics and Mathematics, National Taras Shevchenko University, Academician Glushkov Avenue 6 , KYIV 03127, UKRAINE

E-mail address: myus@univ.kiev.ua

Department of Probability Theory and Mathematical Statistics, Faculty for Mechanics and Mathematics, National Taras Shevchenko University, Academician Glushkov Avenue 6 , KYIV 03127, UKRAINE

E-mail address: corlagon@univ.kiev.ua

Received 1/DEC/2005

Translated by O. I. KLESOV 